\title{
POPULAÇÃ̃O, CENTRALIDADE RELATIVA E MORFOGÊNESE SISTÊMICA EM ÁREAS URBANAS DO ESTADO DE SÃO PAULO *
}

\section{Manoel Tosta BerLinck **}

Uma das preocupações centrais e permanentes das ciências do comportamento humano tem sido a de descobrir e analisar os mecanismos que provocam transformações estruturais em sistemas sócioculturais. Pode-se dizer, até, que as origens próximas dessas ciências coincidem com tal preocupação. Assim, por exemplo, o evolucionismo darwiniano influencia o pensamento de COMTE e SPENCER e leva êste último a declarar que

"é também uma das características dos corpos sociais, como dos corpos vivos, que enquanto aumentam em tamanho, aumentam em estrutura" ${ }^{1}$.

* O autor agradece a colaboração de MARIA Cecília Galli, Maria Tosta Berlinck, ANTônio Gonçalves e Vicente Trevas que participaram na coleta dos dados da pesquisa. Agradece, também, a colaboração de Youssef CoHeN que participou na análise estatística do material empírico. Este trabalho foi possível graças ao apoio de Horácio BERLINCK NeTo e da Escola de Administração de Emprêsas de São Paulo, da Fundação Getúlio Vargas. E desnecessário dizer, entretanto, que qualquer êrro que haja no trabalho é de inteira responsabilidade do autor.

** Professor-Assistente do Departamento de Ciências Sociais da Escola de Administração de Emprêsas de São Paulo, da Fundação Getúlio Vargas. 1 SPENCER, Herbert. The Evolution of Societies, in: Social Changes: Sources, Patterns and Consequences, ETzIoni Amitai e ETzion, Eva. (eds), Nova Iorque, Basic Books, Inc. Publishers, 1964, p. 10 a 15. 
Esta proposição é, mais tarde, revista por DuRKhEIM que demonstra que o simples aumento demográfico de uma sociedade não provoca, necessàriamente, uma transformação em sua estrutura e conclui que

"a divisão do trabalho varia em relação direta com o volume e densidade das sociedades e se progride de maneira contínua no curso do desenvolvimento social é porque sociedades se tornam regularmente mais densas e geralmente mais volumosas". ${ }^{2}$

É lógico que os cientistas sociais do Século XX já conseguiram progredir bastante a partir dos alicerces lançados pelos pensadores acima citados. Assim é que antropólogos como MaUss, RadCliffe-Brown, Malinowski, KROEBER, LINTON, HERSKOVITZ e outros introduziram e desenvolveram a noção de mudança cultural como um dos mecanismos centrais do processo. ${ }^{3}$

Depois da Segunda Guerra Mundial, a preocupação dos cientistas sociais com o processo de desenvolvimento sócio-econômico das colônias européias levou milhares de indivíduos do mundo inteiro a se dedicarem ao problema, e a literatura sôbre o assunto progrediu e multiplicou-se

DURKHeIM, Emile. The Division of Labor in Society, Nova Iorque, The Press, 1964, p. 262.

a Mauss, Marcel. The Gift: Forms and Functions of Exchange in Archaic Societies, Londres, Cohen and West, Ltd., 1966. RAdCLIFFe-Brown, A. R. Structure and Function in Primitive Society, Nova Iorque, The Free Press, 1965. MalinowsKI, Bronislaw. Magic, Science and Religion, Garden City, N. Y., Doubleday and Co., Inc., 1954. Kroeber, A. L. Diffusionism, in: ETZIONI, Amitai è Etzioni, Eva (eds.) Social Change: Sources, Patterns and Consequences, Nova Iorque, Basic Books, Inc., Publishers, 1964, p. 142 a 146. Linton, Ralph. Discovery, Invention and Their Cultural Setting, in: ETzIonI, Amitai e Etzloni, Eva (eds.) Social Change: Sources, Patterns and Consequences, Nova Iorque, Basic Books, Inc., Publishers, 1964, p. 431 a 439. Herskovitz, Melville J. The Process of Cultural Change, in: Linton, Ralph (ed.) The Science of Man in the World Crisis, Nova Iorque, Columbia University Press, 1945, p. 143 a 170. 
fantàsticamente. ${ }^{4}$ Surgiram noções como "sociedade em transição", "sociedade subdesenvolvida", "modernização", "industrialização", "urbanização", "personalidade realizadora", "cultura inovadora" e outras que, muitas vêzes, refletem modelos originais de explicação. ${ }^{5}$

Mas o problema central continua a existir: à medida em que comunidades humanas crescem e perduram, elas demonstram uma tendência a apresentar crescente especialização interna, que nada mais é do que um reflexo do processo geral da progressiva divisão do trabalho. Isto quer dizer, em outras palavras, que comunidades humanas tendem a manifestar progressiva diferenciação estrutural e especialização funcional. À medida que êste processo ocorre, uma rêde mais complicada de partes interdependentes e especializadas constitue um sistema cada vez mais complexo. ${ }^{6}$

Essa tendência geral manifesta-se, entretanto, de maneiras diversas. Enquanto certas comunidades apresentam diferenciação e especialização crescentes, outras comunidades evoluem tão lentamente que, para efeitos de análise, são consideradas pràticamente estáticas. Por outro lado, certas

- Entre a vasta literatura sôbre o assunto, podemos citar:BARRINGER, Herbert H.; Blankstein, George L. e MACK, Raymond W. Social Change in Underdeveloped Areas: A Reinterpretation of Evolutionary Theory, Cambridge, Mass., Schenkman Publishing Col., 1966 BeLSHAw, Cyril S. Traditional Exchange and Modern Markets, Englewood Cliffs, N. J., Prentice-Hall, Inc., 1965. Blumer, Herbert. Industrialization and the Traditional Order, Sociology and Social Research, Vol. 48, n..$^{\circ}$ 2, January, 1964, p. 129 a 138. Boulding, Kenneth E. The Meaning of the 20th Century, Nova Iorque, Harper and Row, Publishers, 1965. DRUCKER, Peter F. The Future of Industrial Man, Nova Iorque, Mentor Books, 1965. Foster, George M. Ttaditional Cultures and the Impact of Technological Changes, Nova Iorque, Harper and Brothers, 1962. HALl, Edward T. The Silent Language, Garden City, N. Y., Dobleday and Co., Inc., 1959. HIRSCHMAN, Albert O. The Strategy of Economic Development, New Haven, Yale University Press, 1958. LewIs, W. Arthur. The Theory of Economic Growth, Homewood, Ill., Richard D. Irwin, Inc., 1955. PolANYI, Karl. The Great Transformation, Boston, Beacon Press, 1967. Rostow, W. W. The Process of Economic Growth, Nova Iorque, W. W. Norton and Co., Inc., 1962. STEWARD, Julian H. Theory of Culture Change, Urbana, University of Illinois Press, 1963.

5 Algumas dessas noções são desenvolvidas pelos autores citados acima.

" WARREN, Roland L. Toward a Reformulation of Community Theory, in: WARren Roland L. (ed.) Perspectives on the American Community, Chicago, Rand McNally and Co., 1966. 
comunidades que são consideradas estáticas, em dado momento de sua história, entram em processo de rápida transformação. Finalmente, certas comunidades, que apresentam transformações intensas durante algum tempo, perdem tal intensidade e entram em um estado de "estagnação".

Quais os mecanismos que levam as comunidades humanas a se comportarem dessas maneiras?

Como já tivemos oportunidade de indicar, as respostas a essa pergunta são várias e complexas mas, por motivos que não nos cabe analisar aqui, têm sido consideradas insatisfatórias para os estudiosos do assunto.

Neste trabalho pretendemos formular e testar uma resposta parcial ao problema. Como tôda resposta parcial, não pretende explicar completamente o fenômeno mas acreditamos que apresente algumas vantagens sôbre diversas das que já existem.

Em primeiro lugar, incorpora - ainda que não reproduza - muitas das formulações teóricas vigentes. Em outras palavras, é mais geral e abstrata do que algumas teorias. Em segundo lugar, parece ser cientificamente mais elegante do que algumas teorias vigentes sôbre 0 assunto. ${ }^{7}$ $\mathbf{E}$, em terceiro lugar, parece possuir maior acuidade do que outras teorias, ou seja, apresenta uma correspondência mais adequada com os fenômenos que pretende explicar. O MODELO TÉ́RICO

O domínio empírico da teoria é um sistema sóciocultural. Ela não tem, portanto, qualquer validade se aplicada a sistemas culturais, biológicos ou de personalidade. ${ }^{8}$

Um sistema sócio-cultural pode ser geralmente descrito como um complexo de elementos relacionados, direta ou indiretamente, em uma rêde causal, de tal maneira que

\footnotetext{
7 Uma teoria é mais elegante do que outra à medida que utiliza um número menor de conceitos para explicar a relação entre fenômenos que também são explicados pela primeira teoria. Vide HARP, John. Some Vignettes on Theory Construction, Ithaca, N. Y., Mimeografado, 1964.

$s$ A distinção entre êsses sistemas é feita em PARsons, Talcott. The Social System, Nova Iorque, The Free Press, 1961.
} 
cada elemento seja relacionado com pelo menos alguns outros, de um modo mais ou menos estável, dentro de um período determinado de tempo. Os tipos particulares de inter-relacionamentos relativamente estáveis de componentes identificados no tempo constituem a estrutura particular do sistema naquele momento, atingindo, dessa forma, uma espécie de "todo" com algum grau de continuidade e limites. ${ }^{9}$

Ocorre, entretanto, que em sistemas sócioculturais processos de troca com o ambiente externo aos seus limites são relativamente constantes. Em outras palavras, ao contrário de outros sistemas naturais que são fechados, os sistemas sócioculturais são abertos.

Sistemas fechados são regidos pela segunda lei da termodinâmica. Tal lei afirma que, em qualquer sistema fechado, uma certa quantidade chamada entropia deve aumentar de maneira a que o processo venha estacionar no momento em que as partes do sistema se encontrem em um estado de equilíbrio. ${ }^{10} \mathrm{~A}$ maximização da entropia em um sistema fechado tende a minimizar as diferenças entre as partes e tende, portanto, a maximizar a desorganização entre as partes do sistema. ${ }^{11}$

A dinâmica dos sistemas abertos, por outro lado, é regida por princípios diferentes dos que regem sistemas fechados. Os princípios fundamentais dos sistemas abertos são, além da entropia, o da equifinalidade e o da informação. ${ }^{12}$

Enquanto que, em qualquer sistema fechado, o seu estado final é inequìvocamente determinado pelas condições iniciais, ou seja, se ou as condiçõos iniciais ou o processo forem alterados, o estado final também será modificado,

- BUCKLEY, Walter. Sociology and Modern System Theory, Englewood Cliffs, N J., Prentice-Hall, Inc., 1967.

10 BertalanfFy, Luwig Von. General System Theory, in: System, Change and Conflict, Nova Iorque, Demerath, N. J., e Peterson, Richard A. (eds) The Free Press, 1967, p. 115 a 130.

13 BertalanfFy, Ludwig Von. Ob. cit.

1:2 Idem, ibidem. 
tal fenômeno não ocorre em sistemas abertos. ${ }^{13}$ Neste caso. o mesmo estado final pode ser obtido a partir de condições iniciais diferentes e através de diferentes maneiras. $E$ isto que se chama equifinalidade sistêmica. ${ }^{14}$

O princípio da informação sugere que partes dos sistemas abertos obtêm, do meio-ambiente externo a êles, os elementos necessários a minimizar a entropia. Como a entropia é uma tendência à desordem e como a informação minimiza a entropia, partes dos sistemas abertos podem aumentar em ordem e organização, à medida que a quantidade de informação que obtêm do meio-ambiente seja maior que a entropia. ${ }^{15}$

Aos processos de transformação que visam a aumentar a ordem e a organização das partes dos sistemas abertos, inclusive entre elas mesmas, pode-se dar o nome de morfogênese. ${ }^{16} \mathrm{O}$ tipo de morfogênese que nos interessa, no momento, é a diferenciação estrutural que pode ser definida como o processo de acôrdo com o qual uma parte do sistema se transforma em duas ou mais partes que passam a funcionar mais eficazmente nesta nova circunstância histórica. As novas unidades são estruturalmente distintas umas das outras, mas, em conjunto, são funcionalmente equivalentes à unidade original. ${ }^{17}$

A diferenciação estrutural é uma das bases da especialização e, portanto, da complexidade sistêmica. Diz-se, portanto, que sistemas cujas partes são relativamente simples, são funcionalmente difusos, ao passo que sistemas cujas partes são complexas, são funcionalmente específicos.

O grau de complexidade sistêmica determina a capacidade de o sistema não só adaptar-se ao meio-ambiente como,

\footnotetext{
Is Idem, ibidem.

14 Idem, ibidem.

1s A noção de meio-ambiente inclui, aqui, outros sistemas com os quais o analisado encontra-se em permanente contato.

16 BUCKLEY, Walter. Ob. cit.

17 Smelser, Neil J. Mechanisms of Change and Adjustment to Change, in Industrialization and Society, Paris, Unesco-Mouton, 1963, p. 32 a 57. Hoselitz, Bert F. e MOORE, Wilbert E. (eds).
} 
também, à sua capacidade potencial em evoluir. ${ }^{18}$ Vimos que a complexidade sistêmica depende da diferenciação estrutural; que esta é um tipo de morfogênese sistêmica e que a morfogênese depende do princípio da informação que, por sua vez, encontra-se em tensão dialética com a entropia, isto é, a predominância da entropia dificulta ou impede a diferenciação estrutural e esta, por sua vez, ocorre se houver predominância da informação no sistema. A complexidade sistêmica depende, portanto, do princípio da informação. Como o princípio da informáção tende a aumentar a ordem e organização das partes e entre as partes do sistema e êstes fenômenos, por sua vez, permitem a manipulação mais eficaz do ambiente, pode-se dizer que sistemas complexos são mais eficazes do que sistemas simples em suas inter-relações com o meio e apresentam, portanto, maior potencialidade de resolver suas "tensões" internas através de mecanismos evolutivos. ${ }^{19}$.

O pressuposto fundamental da presente teoria é, portanto, o de que quanto mais complexo (específico e diferenciado) é um sistema aberto, maior é a sua capacidade adaptativa às tensões internas e externas que ocorrem constantemente em sua existência.

Como, por outro lado, a complexidade de um sistema depende da sua capacidade em desenvolver mecanismos que visam a aumentar a ordem e a organização das partes em si e entre elas, e estas, por sua vez, dependem da capacidade do sistema em processar informações, podemos dizer então que

quanto maior fôr a capacidade de um sistema em processar informação, maior será sua capacidade adaptativa e, portanto, sua passibilidade de diferenciação estrutural.

is Sahlins, Marshall D. e Service, Elman R. Evolution and Culture, Ann Arbor, The University of Michigan Press, 1965.

is Esta proposição é contrária à formulada por SAHLins e Service. Vide: SAhins, Marshall D. e SeRvice, Elman R. Ob. cit. 
Reduzindo êste conjunto de proposições abstratas, válidas para todos os sistemas abertos, ao nivel mais específico dos sistemas sócioculturais, podemos dizer que a questão fundamental em todo sistema dêste último tipo é a existência de uma população que precisa adaptar-se a seu ambiente (o qual é sempre social, ainda que se constitua também de elementos físicos). $\mathrm{O}$ agregado humano que compõe uma população de um sistema sóciocultural apresenta uma interdependência em questões de manutenção, fabricação de utensílios, invenções, promoção do conhecimento, satisfações emotivas e afetivas, e até na aquisição de um conceito de si mesmo. ${ }^{20}$ Esta interdependência é tão evidente que alguns autores descrevem o comportamento humano como um intercâmbio, aplicando a essa palavra um sentido semelhante ao atribuído pelos economistas. ${ }^{21}$

E possível que a interdependência dos indivíduos humanos encontre suas raízes na incapacidade das pessoas para se dedicarem a duas ou mais atividades diferentes simultâneamente. Enquanto que a maioria dos organismos nascem com um conjunto de utensílios que facilita a sua sobrevivência e adaptação, o homem vem ao mundo completamente despido de qualquer um dêles. Seu estado de nudez requer a realização de um certo número de necessidades em um espaço de tempo muito pequeno. Êste fato não apresentaria problemas se ao alcance do homem houvesse quantidades suficientes daqueles materiais de que necessita. Mas não é êsse o caso. Os materiais necessários se distribuem irregularmente no tempo e no espaço. Este problema se resolve, entretanto, na medida em que dois ou mais indivíduos reúnam suas energias através de certo tipo de comportamento cooperativo. Trabalhando juntos,

\footnotetext{
20 Hawley, Amos H. La Estructura de los Sistemas Sociales, in: HAwLey, Amos H. Las Estructuras de los Sistemas Sociales, Madrid, Editorial Tecnos S.A., 1966, p. 65 a 86.

22 Homans, George C. Social Behavior as Exchange, in: The American Journal of Sociology, Vol. LXIII (1958), p. 597 a 606. BLAU, Peter M. Exchange and Power in Social Life, Nova Iorque, John Wiley and Sons, Inc. 1964.
} 
dois ou mais indivíduos podem realizar mais coisas do que se trabalhassem isoladamente, no mesmo intervalo de tempo. $\mathrm{Na}$ medida em que indivíduos cooperam uns com os outros, começa a desenvolver-se uma organização. Ao mesmo tempo, um ambiente progressivamente mais amplo torna-se acessível à organização e maximiza os recursos disponíveis. ${ }^{22}$

Ao contrário, portanto, de qualquer tipo de interdependência - que chamaríamos de estrutura - os indivíduos humanos têm a capacidade da cooperação que promove a existência da organização.

Cada população humana, por sua vez, procura desenvolver a organização que lhe seja mais instrumental em sua adaptação ao meio. Por outro lado, é de se supor que, sempre que se produzam modificações no meio, a população venha buscar uma nova forma de adaptação, ou seja, uma nova organização adequada às novas circunstâncias. ${ }^{23}$

Essa capacidade dinâmica da organização humana depende, entretanto, da capacidade da população em perceber alternativas e as transformações do meio. Depende, em poucas palavras, de sua capacidade em processar informações. Pode-se dizer, portanto, que, em qualquer sistema aberto,

quanto maior fôt a capacidade de uma população pertencente a um sistema sóciocultural em processar informação, maior será a sua capacidade adaptativa e, portanto, sua possibilidade de diferenciação estrutural.

A justificativa desta proposição para sistemas sócioculturais encontra-se na grande capacidade diferencial do homem em se adaptar às condições ambientais quando comparada com a de outros sêres vivos.

2: HAWLEY, Amos H. Ob. cit.

20 Nicolás, Juan Diez Prólogo, in: Hawley, Amos H. Las Estructuras de los Sistemas Sociales, Madrid, Editorial Tecnos S.A., 1966, p. XI a XX. 
Como diz White, o homem não é diferente dos outros animais porque êle se esforça para sobreviver. Segundo êsse autor, o homem difere dos outros animais não em fins, mas em meios. ${ }^{24}$

"Os meios humanos são meios culturais: cultura é simplesmente a maneira de viver do animal humano. $E$, desde que êste meio ou cultura depende da faculdade exclusivamente humana de usar símbolos, a diferença entre o comportamento do homem e de tôdas as outras criaturas não é simplesmente grande, mas básica e fundamental."

A habilidade de utilizar símbolos significa comunicação de idéias; comunicação significa preservação - tradição — e preservação significa acumulação e progresso. ${ }^{25}$

As definições de "símbolo", "comunicação" e "informação" ainda apresentam problemas nas ciências do comportamento humano. ${ }^{26}$ Entretanto, para introduzir alguma precisão nesta construção teórica, vamos definir símbolo como sendo qualquer sinal carregado de sentido. ${ }^{27} \mathrm{Um}$ sinal pode ser verbal ou não verbal. Símbolos, portanto, podem ser verbais ou não-verbais. Os símbolos desenvolvidos pelo homem podem ser agrupados de diversas maneiras. ${ }^{28} \mathrm{O}$ que nos interessa, no momento, é saber que o conhecimento é um conjunto de símbolos, e que difere de outros conjuntos de símbolos na medida em que o definimos como aquilo que é considerado verdadeiro a respeito

\footnotetext{
st White, Leslie A. The Science of Culture, Nova Iorque, Grove Press, Inc., 1949.

25 WhITE, Leslie A. Ob. cit., p. 34.

- Idem, ibidem.

- Newman, John, B. A. Rationale for a Definition of Communication, in: Holt, Rinehart and Winston, 1966, p. 55 a 63 . SMITH, Alfred G (ed.).

Rinehart and Winston, 1966 , p. 55 a 63.

28 WILliams JR., Robin M. American Society: A Sociological Interpretation, Nova Iorque, Alfred A. Knopf, Inc., 1961.
} 
do mundo empírico. ${ }^{29} \mathrm{O}$ conhecimento é, pois, a parte da cultura que possibilita ao homem uma adaptação criadora no meio em que vive. ${ }^{30}$

A informação difere da comunicação na medida em que a primeira envolve a transmissão de conhecimentos. Enquanto que a comunicação é transmissão simbólica, a informação é um tipo de transmissão simbólica que possibilita o aumento do conhecimento. ${ }^{31}$

A comunicação é transmissão de símbolos. Os símbolos transmitidos podem ou não se referir ao mundo empírico. Quando os símbolos transmitidos possuem referência empírica, podem ou não ser considerados como verdadeiros a respeito do objeto a que se referem. Sòmente quando êles são considerados verdadeiros é que a comunicação se transforma em informação.

Quando há informação, aspectos do ambiente anteriormente ignorados passam a ser percebidos e essa percepção é condição necessária para que tal meio seja manipulado. Nas palavras de MCLUHAN, a informação transforma o meio-ambiente em antimeio-ambiente. ${ }^{32}$

Vimos, portanto, que a informação depende da comunicação. Esta, por sua vez, depende da capacidade populacional em transmitir, receber e processar sinais carregados de sentido.

Pode-se dizer, portanto, que

quanto maior fôr a capacidade de ưma população pertencente a um sistema em transmitir, receber e processar símbolos, maior a sua capacidade em transmitir, receber e processar informações.

${ }_{29}$ WILLIAMS JR., Robin M. Ob. cit.

30 Idem, ibidem.

31 MCluHaN, Marshall, The Relation of Environment to Anti-Environment, in: The Human Dialogue: Perspectives on Communication, Nova Iorque; MATson, Floyd W. e Montagu, Ashley (eds.).

32. MCLUHAN, Marshall. Ob. cit. 
Como, por sua vez, a capacidade da população de um sistema em transmitir, receber e processar informações determina a possibilidade de diferenciação estrutural de suas organizações, conclui-se que:

quanto maior fôt a capacidade da população de um sistema em transmitir, receber e processar símbolos, maior a passibilidade de diferenciação estrutural de suas organizações.

O problema teórico central é, portanto, o de se determinarem as condições que levam a população de um sistema a aumentar sua capacidade em transmitir, receber e processar símbolos.

Tanto a transmissão como a recepção de símbolos dependem de canais de comunicação. Diferentes sistemas apresentam quantidades diferentes de canais de comunicação. $E$ de se esperar que quanto maior fôr o número de canais de comunicação de um sistema, maior será a capacidade de sua população em obter e transmitir informações. A essa característica sistêmica vamos chamar de centralidade relativa. ${ }^{33}$

Voltando, agora, à proposição hipotética anteriormente formulada, pode-se dizer que

quanto maior a centralidade relativa da população de um sistema, maior a capacidade de diferenciação estrutural de suas organizações.

O processamento de símbolos, por sua vez, é uma atividade eminentemente organizacional. No caso dos sistemas sócioculturais, o processamento de símbolos depende da quantidade de diferentes organizações que existem no sistema, pois é a partir da ação cooperadora grupal que símbolos podem ser manipulados e atividades exercidas, a partir do processamento de tais símbolos.

$\overline{\text { Idem, ibidem. }}$ 
Vimos, por outro lado, que à medida que indivíduos cooperam, um ambiente, progressivamente mais amplo, torna-se acessível.

Portanto, a quantidade de organizações e seu tamanho dependem do tamanho da população existente em um sistema sóciocultural ${ }^{34} \mathrm{Em}$ outras palavras,

quanto maior a população de um sistema sóciocultural, maior a possibilidade de diferenciação estrutural de suas organizações.

Cremos, entretanto, como DuRKheim, que a população de um sistema é condição necessária, mas não suficiente para a ocorrência de diferenciação estrutural. ${ }^{3 \check{5}} \mathrm{Em}$ outras palavras, acreditamos que em sistemas com populações semelhantes, os que possuem maior número de canais de comunicação devem apresentar maior diferenciação estrutural.

A proposição hipotética básica dêste quadro teórico é, portanto, a seguinte:

Controlando-se o tamanho das populações de sistemas sócioculturais, quanto maior o número de canais de comunicação do sistema, maior a possibilidade de diferenciação estrutural de suas orǵanizações.

\section{O TESTE DO MODELO}

O teste da teoria acima formulada foi realizado em áreas urbanas do Estado de São Paulo. Para tanto, adotamos a divisão do estado em regiões apresentada pelo IBGE. ${ }^{26}$ Em seguida, passamos a analisar quatro dessas regióes, a saber: Norte, Sul, Leste e Vale do Paraíba. Cada uma dessas regiões é dividida em municípios. Cada município

\$4 Young, Frank W. A Proposal for Cooperative Cross-Cultural Research on Intervillage Systems, Human Organization, vol. 25, primavera de 1966, p. 46 a 50.

${ }_{85}^{46}$ Tal fato decorre da capacidade restrita do homem em participar de diferentes grupos sociais.

su DURKHEIN, Emile. Ob. cit. 
possui uma área urbana. A área urbana de cada município foi considerada como sendo um sistema aberto. Para cada área urbana foi obtida a sua população. ${ }^{37} \mathrm{Em}$ seguida, a partir de informações obtidas através das Listas Telefônicas Amarelas, construíram-se dois índices para cada área urbana:

- o primeiro é a soma do número de canais de comunicação existentes em cada uma das áreas urbanas. Considerou-se como canal de comunicação todo meio dedicado direta ou indiretamente à transmissão de símbolos; ${ }^{38}$

- o segundo índice é a soma de diferentes tipos de organizações formais existentes em cada área urbana. ${ }^{39}$ Note-se que aqui não se trata de quantidade mas de diferentes tipos de organizações. Assim, se em uma determinada área urbana existem dez escritórios de advocacia, o valor adicionado no índice é um. Para se evitar a contaminação no teste, eliminaram-se neste índice tôdas as organizações computadas no primeiro.

A primeira parte do teste da hipótese foi feita através do cálculo de correlação de postos entre a população, os canais de comunicação e as organizações formais das áreas urbanas dos municípios de cada região considerada. ${ }^{40}$

${ }^{37}$ O IBGE divide o estudo em seis áreas, a saber: Norte, Sul, Leste, Oeste, Vale do Paraíba e Litoral Sul.

ss Os dados populacionais são os do censo de 1960. Vide Instituto Brasileiro de Geografia e Estatística: Anuário Estatístico, Rio de Janeiro, 1961.

3v Foram as seguintes as organizações consideradas como canais de comunicação: aeroportos, associações em geral, associações comerciais, bibliotecas, cinemas, clubes, organizações dedicadas a diversões, escolas, gráficas, hotéis, igrejas e agremiações religiosas, jornais, lojas de livros, mercados, emprêsas de navegação aérea, linhas de ônibus, pontos de automóveis de aluguel, pontos de ônibus de aluguel, emprêsas de propaganda, estações de rádio, distribuidores de revistas, sindicatos, emprêsas de táxi aéreo, tipografias e emprêsas de transportes pesados urbanos, interurbanos e interestaduais.

to E importante que se reconheça que tais indices são medidas grosseiras, pois a fonte de onde foram retirados os dados não é completa. Pode-se supor, entretanto, que o possível êrro que existe devido à qualidade da fonte seja neutralizado por ocorrer em todos os centros urbanos de maneira semelhante. 
Na Tabela I encontram-se os resultados dessas correlações.

TABEla I: Cotrelação de Postos entre População Utbana, Canais de Comunicação e Divisão do Trabalho em Quatro Regiōes do Estado de São Paulo

Norte

\begin{tabular}{|c|c|c|c|}
\hline & $\begin{array}{c}\text { Popu- } \\
\text { lação }\end{array}$ & $\begin{array}{c}\text { Comu- } \\
\text { nicação }\end{array}$ & $\begin{array}{c}\text { Divisão } \\
\text { do } \\
\text { Trabalho }\end{array}$ \\
\hline $\begin{array}{c}\text { Popu- } \\
\text { lação }\end{array}$ & 0,53 & 0,86 \\
\hline $\begin{array}{c}\text { Comu- } \\
\text { nicação }\end{array}$ & $-1,89$ \\
\hline $\begin{array}{c}\text { Divisão } \\
\text { do } \\
\text { Trabalho }\end{array}$ & & & \\
\hline
\end{tabular}

Leste

\begin{tabular}{|c|c|c|c|}
\hline & $\begin{array}{c}\text { Popu- } \\
\text { lação }\end{array}$ & $\begin{array}{c}\text { Comu- } \\
\text { nicação }\end{array}$ & $\begin{array}{c}\text { Divisão } \\
\text { do } \\
\text { Trabalho }\end{array}$ \\
\hline $\begin{array}{c}\text { Popu- } \\
\text { lação }\end{array}$ & 0,32 & 0,81 \\
\hline $\begin{array}{c}\text { Comu- } \\
\text { nicação }\end{array}$ & Divisão \\
\hline $\begin{array}{c}\text { do } \\
\text { Trabalho }\end{array}$ & 0,74 \\
\hline
\end{tabular}

Sul

\begin{tabular}{|c|c|c|c|}
\hline & $\begin{array}{c}\text { Popu- } \\
\text { lação }\end{array}$ & $\begin{array}{c}\text { Comu- } \\
\text { nicação }\end{array}$ & $\begin{array}{c}\text { Divisão } \\
\text { do } \\
\text { Trabalho }\end{array}$ \\
\hline $\begin{array}{c}\text { Popu- } \\
\text { lação }\end{array}$ & 0,49 & 0,82 \\
\hline $\begin{array}{c}\text { Comu- } \\
\text { nicação }\end{array}$ & $-1,88$ \\
\hline $\begin{array}{c}\text { Divisão } \\
\text { do } \\
\text { Trabalho }\end{array}$ & - & \\
\hline
\end{tabular}

Vale do Paraíba

\begin{tabular}{|c|c|c|c|}
\hline & $\begin{array}{c}\text { Popu- } \\
\text { lação }\end{array}$ & $\begin{array}{c}\text { Comu- } \\
\text { nicação }\end{array}$ & $\begin{array}{c}\text { Divisão } \\
\text { do } \\
\text { Trabalho }\end{array}$ \\
\hline $\begin{array}{c}\text { Popu- } \\
\text { lação }\end{array}$ & 0,70 & 0,90 \\
\hline $\begin{array}{c}\text { Comu. } \\
\text { nicação }\end{array}$ & 0,80 \\
\hline $\begin{array}{c}\text { Divisão } \\
\text { do } \\
\text { Trabalho }\end{array}$ & & \\
\hline
\end{tabular}

Os resultados obtidos indicam que, de maneira geral, a relação entre população e comunicação é bem menor que as relações entre população e divisão do trabalho e canais de comunicação e divisão do trabalho. Os resultados sugerem, portanto, que o crescimento da população não corresponde, necessàriamente, a um aumento do número de canais de comunicação. Por outro lado, tanto o aumento da população como o aumento dos canais de comunicação 
correspondem a um aumento da divisão do trabalho, no universo aqui analisado. Tais resultados confirmam, dessa forma, as hipóteses formuladas no quadro teórico.

A Tabela II apresenta correlações de postos entre canais de comunicação e divisão do trabalho, controlando-se por tamanho da população urbana do universo analisado.

TABELA II: Correlação de Postos entre Canais de Comunicação e Divisão do Trabalho em Cidades de Mesma População, em Quatro Regióes do Estado de São Paulo

\begin{tabular}{|c|c|c|c|c|}
\hline & Norte & Sul & Leste & $\begin{array}{c}\text { Vale } \\
\text { do } \\
\text { Paraíba }\end{array}$ \\
\hline Cidades de 1 a 3.000 habitantes & 0,66 & 0,63 & 0,67 & 0,44 \\
\hline Cidades de 4 a 6.000 habitantes* & 0,88 & 0,86 & 0,56 & - \\
\hline $\begin{array}{c}\text { Cidades de } 10.000 \text { ou mais habi- } \\
\text { tantes..................... }\end{array}$ & 0,98 & 0,93 & 0,71 & 0,77 \\
\hline
\end{tabular}

* O número de cidades de 4 a 6.000 habitantes, no Vale do Paraíba, é muito pequeno, impossibilitando, portanto, o cálculo de uma correlação de postos

Os resultados apresentados na Tabela II sugerem que, em centros urbanos de população semelhante, o aurnento do número de canais de comunicação está relacionado com um aumento de diferentes organizações formais, o que vem confirmar a hipótese formulada. Ocorre, entretanto, que tal relação é muito mais forte em cidades de $\mathbf{1 0 . 0 0 0}$ habitantes ou mais do que em cidades com menos de ... 10.000 habitantes.

Em outras palavras, os resultados apresentados na Tabela II sugerem que a relação entre canais de comunicação e divisão do trabalho aumenta consideràvelmente na medida em que a população de centros urbanos aumenta, o que vem confirmar a hipótese formulada. E possível que tal diferença seja explicada pela diferença da própria natureza das comunicações entre os membros de centros urbanos 
pequenos e grandes. Enquanto, em cidades pequenas, o processo de interação se dá através da comunicação direta, face a face, onde os indivíduos utilizam a voz humana para transmitir símbolos, nas cidades grandes o mesmo não ocorre. Nesta situação, os canais impessoais de comunicação, como rádio, telefone, etc., são muito mais utilizados. Como, por outro lado, foram êsses canais impessoais os utilizados na pesquisa, a correlação entre êles e a divisão do trabalho em cidades pequenas apresenta uma variância muito grande.

Só uma pesquisa mais detalhada poderia resolver esta dúvida. Ocorre, entretanto, que mesmo que isso fôsse verdade, não haveria um aumento dos índices de correlação obtidos. Em outras palavras, o tipo de interação que se dá entre os indivíduos da população de uma cidade pequena não está ìntimamente relacionado com um aumento da diferenciação estrutural. Ora, se isso fôr verdade, podese concluir que não basta que indivíduos se comuniquem E necessário haver troca de informações, nesse processo de interação, para que haja diferenciação estrutural.

\section{RESUMO E CONCLUSÕES}

min

Este trabalho objetivou mostrar que a crescente diferenciação estrutural de organizações pertencentes a sistemas sócioculturais depende do tamanho de sua população e da capacidade dêsse agregado em receber, transmitir e processar informações.

O teste empírico da construção teórica, realizado em áreas urbanas do Estado de São Paulo, demonstrou que a diferenciação estrutural varia diretamente, tanto com a variação da população, como com a variação da quantidade de canais de comunicação que são indicadores da capacidade do agregado em receber, transmitir e processar informações.

Quando, entretanto, se controla o tamanho do agregado populacional, verifica-se que há, não só, uma íntima rela- 
ção direta entre a variação dos canais de comunicaçâu e a complexidade estrutural do sistema, como se constata que essa relação é maior em sistemas cuja população é maior.

A explicação encontrada é a de que, não só o tamanho da população possibilita uma maior diferenciação estrutural, mas a de que o próprio tipo de comunicação - mais orientada para a transmissão de informações, nas áreas urbanas maiores - é que causa tal diferença.

A teoria é, assim, confirmada, ainda que o teste empírico tenha sido grosseiro e requeira pesquisa mais cuidadosa. Entretanto, pode-se afirmar que os resultados obtidos são bastante animadores.

A importância da teoria está em seu requinte científico e nas implicações que ela contém para o desenvolvimento de áreas subdesenvolvidas. ${ }^{41}$

Se tal teoria fôr aceita como verdadeira, pode-se deduzir que a primeira condição necessária para o desenvolvimento sócio-econômico de áreas relativamente despovoadas é o aumento de sua população. As dificuldades que o desenvolvimento enfrenta aumentam na medida em que o tamanho da população da área diminui.

Pode-se concluir, também, que o desenvolvimento de áreas relativamente povoadas pode ser acelerado à proporção que se aumenta o número de canais de comunicação na área.

$\mathrm{O}$ aumento de canais possibilita um fluxo maior e mais amplo de informações que, por sua vez, possibilitam não só a percepção de novos aspectos do meio como, também, a sua manipulação mais eficiente para a satisfação das necessidades e desejos humanos.

a $O$ autor deseja agradecer os conselhos estatisticos de NAGIB FEREs, professor da Universidade de São Paulo e de Fábio Luís MARIotTo, professor-assistente da Escola de Administração de Emprêsas de São Paulo, da Fundação Getúlio Vargas. Entretanto qualquer êrro de interpretação ou de cálculo que, porventura, exista nesta análise é de inteira e única responsabilidado do autor dêste trabalho. 
Essa situação pode ser observada na tão falada revolução das expectativas que se verificou nos países do terceiro mundo, depois da Segunda Guerra Mundial.

A revolução das expectativas foi resultado da quebra do isolamento em que se encontravam as populações dos países subdesenvolvidos. Mas o rompimento de tal isolamento só foi possível graças às profundas transformações ocorridas nos meios de comunicação. ${ }^{42} \mathrm{~A}$ introdução ou a expansão dos canais de comunicação permitiram que tais populações obtivessem informações a respeito do estilo de vida das sociedades desenvolvidas e percebessem que a sua pobreza não dependia de fôrças ou fenômenos incontroláveis, mas era decorrência de condições históricas concretas que, eventualmente, poderiam ser controladas. ${ }^{4: 3}$ É de se esperar, portanto, que, com a introdução e a expansão dêsses canais, as sociedades subdesenvolvidas venham a explorar mais adequadamente o meio em que vivem, a fim de promoverem melhores condições para sua vida.

\footnotetext{
12 Cremos que o desenvolvimento sócio-econômico pode ser considerado como um efeito concomitante da complexidade sistêmica.

" LERner, Daniel. The Passing of Traditional Society, Nova Iorque, The Free Press, 1964, SChramm, Wilbur. Mass Media and National Development, Stanford, California, Stanford University Press, 1964.
} 